\title{
Wisdom of the Crowd within Enterprises: Practices and Challenges
}

\author{
Mahmood Hosseini ${ }^{1}$, Jack Moore, Malik Almaliki, Alimohammad Shahri, Keith \\ Phalp, Raian Ali ${ }^{1,}$ \\ Bournemouth University, UK
}

\begin{abstract}
The Wisdom of the Crowd advocates that decisions collectively made by a diverse crowd could be better than those made by an elite group of experts. The Wisdom of the Crowd puts preconditions on this to work correctly. This concerns the diversity of the crowd, their independence from each other, their decentralisation, and the methods of aggregating their distributed knowledge and forming collective decisions. Although the concept is inspiring, its interpretation and conduct differ significantly amongst enterprises, especially with regard to the culture and style of management. In addition, we still lack reflections on how the Wisdom of the Crowd worked in the practice of modern enterprises. To address this lack of knowledge, this paper conducts an empirical study following a mixed method approach involving 35 senior managers coming from 33 different industries in the UK. In the first phase we interview eight managers and, in the second, we confirm and enhance the results by a survey consisting of open-ended questions and involving 27 other managers. The results shed light on the current practice of the Wisdom of the Crowd in several UK enterprises, which can inform the analysis and design of future software tools meant to aid this emerging decision-making mechanism.
\end{abstract}

Keywords:

Wisdom of the Crowd, Collective Intelligence, Decision Making

Email addresses: mhosseini@bournemouth.ac.uk (Mahmood Hosseini), raliabournemouth.ac.uk (Raian Ali) 


\section{Introduction}

The Wisdom of the Crowd (WoC) advocates that, for certain kinds of problems, the collective decisions of a large group of individuals are often better than those taken by an elite group of experts (Surowiecki, 2005). The reliance on the crowd to solve problems and take decisions has a long history in social and political science, including the foundations of modern democratic systems and its composite and emerging forms like the urban governance (Pierre, 2000). The concept, coined as the WoC, has gained attraction with the advent of the technology age, which made it more feasible not only for large scale enterprises, e.g. parliaments and other governmental institutes, but also for small and medium scale enterprises and smaller scale problems. For example, it is possible now to see a small design company consulting staff and clients about the new logo or the new website style and features.

In order for the WoC to yield good results, there are a number of aspects that need to be set up (Surowiecki, 2005; Surowiecki, et al., 2007). The first is about the diversity in the crowd, where diversity is generally needed to generate valid and holistic solutions. The second is about independence amongst the members of the crowd, where dependence means suppression of individual perspectives, sometimes subconsciously, e.g. when influential people drive the crowd. The third is about decentralisation in the sense of specialisation where members or groups work on parts of the problem and provide local knowledge, i.e. divide and conquer. The fourth is about the suitable aggregation of that local knowledge to form a holistic solution. These four pillars were often studied as general principles without concretising and delving into the details. Thus, customisation to the problem domain typically requires further research.

In academia, the WoC has been researched as a mechanism to solve a wide range of problems. This includes the harnessing of the power of the crowd to contribute enterprise knowledge (Oinas-Kukkonen, 2008), in supporting marketing (Kozinets, et al., 2008), in content generation (Fuxman, et al., 2008), in prediction and forecasting (Jin, et al., 2010), in election forecasting in politics (Murr, 2011), and in e-commerce and collaborative shopping (Curty and Zhang, 2011). Certain studies paid effort on subtle aspects of the WoC such as the effect of coordination strategies and also social influence amongst the crowd on the quality of outcome (Kittur and Kraut, 2008; Lorenz, et al., 2011). The underlying reasons for this growth of research relate to the facilities Web 2.0 provides and the proliferation

of social networks, which made online communication both effective and widely used. Surprisingly, the majority of research focused on the final stages of the WoC, mainly on deducing collective decisions from de facto social networks or 
artificial agents. Little attention has been paid to the forward engineering of platforms and social networks expressly tailored to the WoC and to meet its principles of diversity, independence, decentralisation and aggregation.

Knowledge acquisition is a bottleneck problem in enterprises (Motta, 2013). The collection of knowledge is a preliminary step to quality enterprise modelling and decision making. This is typically a collaboration amongst clients, staff, management and systems analysts (Barjis, et al., 2009). Broadening the volume and expertise of the set of stakeholders involved in the knowledge acquisition would help better quality knowledge (Vukovic, 2009). For modern enterprises to cater for the velocity and diversity of changes in their social and economic context, the WoC seems to be a practical and realistic alternative to the centralised knowledge acquisition and decision making approaches especially with the presence of the new technology of social networks and mass communication.

In spite of the promising nature of the WoC for modern enterprises, we still lack the knowledge on how the concept is perceived and applied in practice. Also, being mainly a social concept, the WoC would highly be subject to multiple interpretations in different enterprise cultures. In this paper, we investigate how the WoC is perceived and practised in several United Kingdom enterprises. In doing so, we also aim at reinforcing the meaning of the main pillars of the WoC and identifying questions to ask when designing automated tools to support the WoC. The study consists of an exploratory qualitative interview-based phase and a quantitative survey-based phase, and it involves 35 managers from 33 different UK enterprises.

The paper is structured as follows. In Section 2, we present our research method and the design of our study. In Section 3, we report our findings on the current practice of the WoC and its challenges. In Section 4, we elaborate on our findings and discuss the threats to validity of our study. In Section 5, we present conclusions and future work.

\section{Methodology}

To investigate how the $\mathrm{WoC}$ is applied in enterprise decision-making and problem solving, we conducted an empirical study using a sequential exploratory mixed method. The first phase of the study was qualitative, where we interviewed eight managers from eight UK enterprises in various domains. The insights we obtained were then confirmed and enhanced in the next phase, which was quantitative and where we surveyed a larger sample of 27 senior managers working in different enterprises and domains. The questions in the interview and, consequently, those in the survey were centred on the four principles of the WoC as defined 
in (Surowiecki, 2005): diversity, independence, decentralisation and aggregation. The interview and survey questions can be found at http://goo.gl/xQz7DH.

\subsection{Qualitative Phase}

The qualitative phase took two months to complete, during which the following took place. The interview questions were designed based on the concept of WoC and its four pillars and the interview participants were selected. This was mainly done in March 2014 (cf. Section 2.1.1). Then, all the interviews were performed and their results were collected during March and April 2014 (cf. Sections 2.1.2 and 2.1.3). Finally, the content analysis of the interviews and the design of the questionnaire for the online expert survey were performed during April 2014 (cf. Section 2.1.4).

\subsubsection{Interview Participants.}

In order to gather insights and information on how the WoC is being currently practised in enterprises, we first interviewed a select set of eight managers. We followed the purposeful sampling method, proposed by Creswell (Creswell and Clark, 2007), in which participants were selected through the understanding that they usually apply the concept of the WoC in their enterprise. The maximum variation sampling technique (Patton, 2005) was also used to ensure participants diversity. We interviewed seven senior managers with a minimum of 10 years of experience and one junior manager with two years of experience. We chose the participants from eight different industries in the UK, namely photography, web development, higher education, environmental and sustainability projects management, pharmaceutical research, network and infrastructure, publishing, and charity organisation.

\subsubsection{Conduct of the Interviews.}

The qualitative interviews were carried out in two separate formats. Six of the eight participants were interviewed face to face, whilst the other two participants were interviewed online. Each participant was invited via e-mail. At the beginning of each interview, each participant was given a consent form to sign agreeing with the terms and conditions of the interview. Then an introduction script and examples were given to ensure we got a shared understanding of the concept of the WoC, especially that managers apply the concept sometimes under different names. This was to ensure that the interviewees were introduced to the concept of WoC and its four pillars before the interview started. Finally, the core questions of the semi-structured interviews took place. During every interview, we sometimes changed the order of the questions with respect to the answers we obtained from 
the managers to keep the natural flow of the information. Therefore, instead of going sequentially with the interview questions in our semi-structured interviews with the managers, we chose to refresh the minds of interviewees to recall their experiences by asking from our list of general purpose questions from time to time.

\subsubsection{Qualitative Data Collection.}

The interviews were all audio-recorded and later transcribed word for word. The average time for an interview was 34 minutes, with the shortest interview being 21 minutes and the longest interview being 47 minutes. Overall 4 hours and 29 minutes of interview data were collected and analysed. The stated amount of time was what we spent on the main questions depending on the answers and does not cover the times spent for greetings, explanations of the $\mathrm{WoC}$ and its four pillars, etcetera. However, considering that managers are typically busy, an average of 34 minutes for the core part of the interview is relatively a generous time especially that the answers were generally succinct and direct to the point without redundancy. They were insightful enough for us to draw conclusions on them and then confirm or reject them via the expert survey with open-ended questions as well.

\subsubsection{Analysis of Qualitative Data.}

For qualitative data analysis, a thorough reading through all transcripts was carried out one by one. Whilst reading over each participant's response to the same question, themes of recurring data were recorded in a code template that had been designed to record trends in responses. Once the text for each response had been coded, the most common trends presented themselves. These common trends were then translated to questions for the quantitative phase, the survey, which included a larger sample of participants for confirmation and enhancement purposes. Two researchers conducted the qualitative phase and a third researcher checked the coding and the results to ensure accuracy. The designed questionnaire also underwent multiple revisions by two researchers to ensure the quality and readability of the questions, and to ensure the points made in the interviews are addressed accordingly, while the questionnaire is not too long to discourage prospective participants. Then the questionnaire was sent to three participants, with managerial experience, just as a trial before sending it to the managers. The comments made by these participants also led to further refinement of the questionnaire. 


\subsection{Quantitative Phase}

The quantitative phase took two months to complete, during which the following took place. The expert survey participants were called for their participation by sending emails which took place in April and beginning of May 2014 (cf. Section 2.2.1). Then, the expert survey was conducted during May 2014, during which the invited managers filled an online questionnaire related to our study of the WoC, and the results were collected (cf. Sections 2.2.2 and 2.2.3). Finally, the results of the online expert survey were analysed during May 2014 (cf. Section 2.2.4).

\subsubsection{Questionnaire Participants.}

The follow up to the qualitative phase was an expert survey. Each participant was sent an e-mail asking if they would like to participate in the survey. This was done as an invitation only survey to ensure that answers are collected from managers only. 27 managers responded positively and completed the questionnaire. The participants had 154.5 years of experience in management roles in total, with an average of 5.7 years. Table 1 summarises the list of sectors of the companies of the managers who participated in the survey.

Table 1: The sectors represented by the companies

\begin{tabular}{|l|c|l|c|}
\hline \multicolumn{1}{|c|}{ Industry } & No. & \multicolumn{1}{c|}{ Industry } & No. \\
\hline Software services & 2 & School education & 2 \\
\hline Car manufacturing & 1 & Marketing & 1 \\
\hline Regional council & 2 & Public relations & 1 \\
\hline Government & 1 & Charity & 3 \\
\hline University body & 2 & Retail & 2 \\
\hline Catering & 1 & Business services & 9 \\
\hline
\end{tabular}

\subsubsection{Conduct of the Questionnaire.}

At the beginning of the questionnaire (cf. http://goo.gl/xQz7DH), a cover sheet explaining the concept of the WoC was issued with links available for participants to look at for further clarification on the topic. This was performed to make sure that the participants have a refreshed, comprehensive understanding of the topic at hand, especially because our interviews indicated that managers use this concept on a daily basis, but under different names such as participatory decision making, collective decision, collaborative problem solving, and so on. Participants were then asked to fill out text boxes explaining their managerial 
level and their experience with the WoC. Five matrix tables were issued in the survey for participants to fill out, consisting of the statements deduced from the qualitative phase, along with optional text entry boxes for additional comments. The questions in the questionnaire were on a five-level Likert scale reflecting the degree of the managers' agreement or disagreement with the statement. These questions were designed to confirm and enhance the insights we obtained from the qualitative phase. The survey contained 49 questions, classified into five main questions.

\subsubsection{Quantitative Data Collection.}

The quantitative research questions were mainly based on a matrix table with text entry boxes to add comments and further clarifications. The questions were devised and distributed using Qualtrics. The average time a participant took to complete the questionnaire was 20 minutes.

\subsubsection{Analysis of Quantitative Data.}

The answers we obtained from the participants in the survey were analysed through Qualtrics descriptive statistical features and tools. We also performed a content analysis of the comments left by the participants in the open-ended questions, which added more meaning to their quantitative answers.

\section{Findings}

In this section, we present the results of our study. These results were firstly deduced from the interviews and then confirmed via the questionnaire, which also allowed adding comments and clarifying the answers. The complete analysis can be found at http://goo.gl/xQz7DH. The mind maps illustrated in Figures 1 to 5 represent our findings which are stated and reflected upon throughout the following subsections. The presented concepts in the mind maps are either presented by the subsections titles and the bullet-points, or these concepts can be found in managers' viewpoints which are stated in different parts of the same subsection.

\subsection{Current Practice of the Use of the WoC}

We first investigated the current practice and perceptions of the WoC in order to have a holistic view on how the concept is being utilised in today's business environments, mainly in the UK. We asked the managers to describe when and how they use the WoC. Figure 1 shows a mind map of our findings. The concept of the WoC was regularly used when one or more of the following situations took place: 


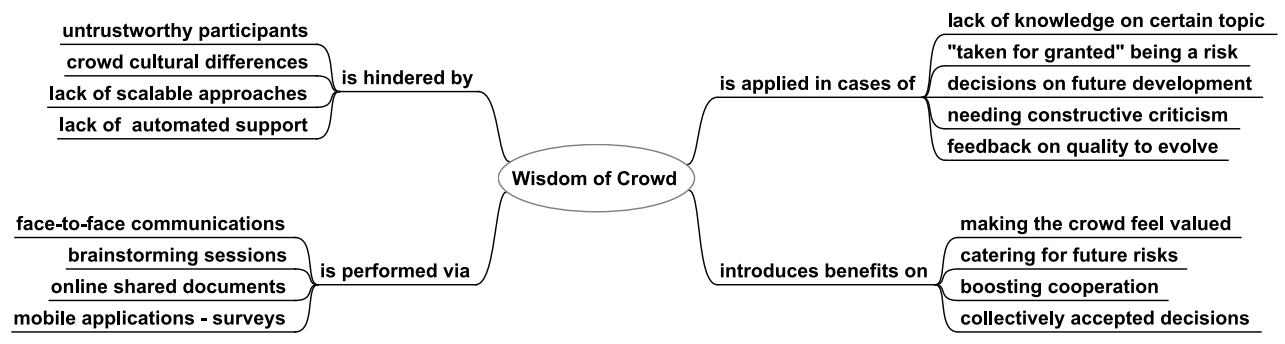

Figure 1: Mind map for the use of the WoC

- When there is a lack of knowledge on certain subjects: Managers indicated that they use the WoC in situations where there is a need for a more or better knowledge and experience regarding certain subjects. In particular, they used the WoC at the concept phase of projects to allow the crowd "to coordinate and focus themselves to meet the common goal and set objectives for the project". This means that sometimes the required knowledge, expertise, or experience may not be present at the managerial level, or even inside an enterprise, and that tapping into the $\mathrm{WoC}$ is a feasible solution to overcome such deficiencies.

- When "taken for granted" is risky: Managers mentioned that they use the WoC to involve independent people alongside experts "to ensure clarity of thought" and to make sure that they can "see the wood for the trees". This means that people who are too involved in the business may sometimes fail to analyse their domain clearly. In such situations, the WoC helps to remove this obstacle by obtaining fresh external perspective or at least inquiring what is taken for granted by the experts within an enterprise.

- When deciding on future development: The WoC was utilised in the "vision days" and action-planning sessions "to help team members feel more attuned" with strategic and operational targets of the enterprise. The WoC here means shared strategic decisions where responsibility is high and consensus amongst stakeholders is a key.

- When constructive criticism is needed: Managers stated that utilising the WoC could give "a balance to our designs" and could also highlight "areas of weakness that we ourselves may not have picked up on". For example, parents could state their opinions on what style of education would better 
fit their children and therefore could alter school decisions as a result. This implies the need for new skills from teachers and that there are particular areas of weaknesses that may require improvement. It can also validate some of the school's assumptions about the nature of students and what teaching methods would be the best for specific subjects.

- When feedback is needed to improve and sustain quality: Feedback differs from constructive criticism as it covers more aspects, such as praising an idea, statement of facts, etcetera. Managers mentioned the use of the WoC during the process of product enhancement, utilising the crowd consisting of their clients and ex-clients for their feedback received over a period of time in various ways. It was deemed important "to gain feedback and listen to everyone involved", because managers admitted that they "may not be the most experienced or qualified to reach a decision on a certain aspect of a project". Using this method they could always incorporate such feedback into enhancement procedures and make changes and sustain quality.

In spite of these benefits, managers also mentioned some downsides of the WoC mainly for two reasons. The first reason is that it might allow for untrustworthy participants to take part is the WoC process, which might result in harm to the business or its related activities. Some managers preferred "to rely on the wisdom of a few trusted sources rather than the general view", when the subject could introduce dishonest participation and when it is critical. This issue has been discussed in details in (Baeza-Yates, 2014). The second reason is that there might be some "cultural issues" amongst the crowd that might make the utilisation of the WoC "less effective". Although diversity in the skills and background knowledge is a desirable feature of the crowd, diversity in the communication styles and the value given to the cooperation and liberty principles could be problematic.

In relation to the process and medium used for applying the $\mathrm{WoC}$, it appears it is mainly based on giving a short presentation, online or in-person, to introduce the issue followed by face-to-face meetings, brain storming sessions, and online shared documents. Managers agreed that this is not often a scalable approach. This limitation sheds light on the need for more automated support to conduct the WoC in a business environment, as we will discuss more elaborately in Section 4. Other managers indicated the use of mobile applications to allow the crowd to provide knowledge in real context and real time and also to reach a wider set of the crowd. The common method is based on simple surveys. These apps can only be of a small scale and allow relatively simple studies requiring limited input and 
limited interaction amongst the members of the crowd.

Besides solving problems, managers indicated that applying the WoC improves staff and clients' attitude and membership feelings towards the enterprise. This includes:

- Giving the crowd a voice to make them feel valued: applying the WoC can positively affect the feeling of partnership of staff and clients with the enterprise and therefore "each person feels that they are a valued member of the company".

- Catering for future risks: Using the WoC allows an enterprise "to ensure all the project risks are catered for", not only those risks related to the development of the product but also those risks related to how the staff and clients would like to participate in the development process and operate the management decisions. Enterprise risk management costs are significant and most risks could be avoided when collaboration is high (Nocco and Stulz, 2006). The WoC increases the feeling of partnership and ownership so that the crowd would become more motivated to collaborate.

- Boosting cooperation and its value: Utilising the WoC boosts the value of cooperation, which would then become a general principle and work style without the need for pre-set mechanisms for it. For example, managers believed that "Working with trust and cooperation where everyone is valued appears to lessen the need for a manager or supervisor because the team works well together".

- Providing collectively accepted decisions: Managers may come with smart solutions which saves effort and money, but such solutions may not necessarily be seen positively by the staff and clients. On the other hand, when managers "give equal weight to the opinions expressed by each member of the team, regardless of their experience, and collectively agree which is the best to take forward", they come with solutions that are more accepted by the crowd, and this also further increases the experience and loyalty of the staff and clients.

\subsection{Diversity: The First Principle of the WoC}

Diversity in the crowd is an essential principle for a correct implementation of the WoC (Surowiecki, 2005). A small group of people are susceptible to a few biased individuals imposing their unjustified influence over the rest of the group. However, bigger, more diverse groups of people are absent of such vulnerabilities 


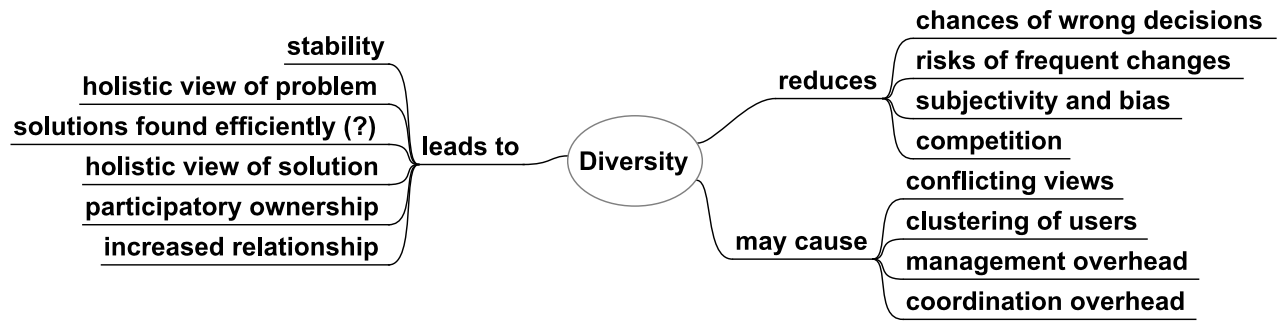

Figure 2: Mind map for Diversity in the WoC

(Surowiecki, et al., 2007). We asked the managers to tell us how they view diversity when they apply the $\mathrm{WoC}$ in their enterprises. Figure 2 shows a mind map of our findings. The question mark (?) in this and following mind maps is to denote that there was a debate on the concept although the majority advocated it.

\subsubsection{Stability.}

Managers stated that diversity in the crowd helps stability in taken decisions and reduces the risk of having frequent changes. Diversity means that the decision has taken into account the different perspectives and possible scenarios. It also means that it accommodates the different natures and roles of those who will be involved in operating the decisions and thus means that frequent changes are avoided.

\subsubsection{Holistic view of the problem.}

Managers agreed that diversity also leads to a more holistic view of the problems in the enterprise. Diversity allows the incorporation of various levels and domains of expertise and helps reducing subjectivity and bias. Diversity means that the definition of the problem is not only done by powerful people but also by those who are in the lower levels of the pyramids and who typically add perspectives less visible to managers.

\subsubsection{Efficient solution finding process.}

Finding solutions for complex problems in enterprises can be costly and timeconsuming. Diversity in the crowd can help enterprises in finding these solutions at low costs. It also allows solutions that would otherwise be missed. However, high diversity, especially when decisions have major effects, leads to conflicting 
views and requires moderations. Given this trade-off, managers still agreed that dealing with conflicts at this stage is better than seeing them when enacting a decision taken by an elite group of experts.

\subsubsection{Holistic view of the solution.}

Diversity was also seen as a way to aid a complete information acquisition. Diversity helps reaching not only a holistic view of the problem, but also aids the completeness of solution knowledge and possible consequences on the various sectors of staff and clients.

\subsubsection{Participatory ownership.}

Diversity also helps people to take ownership of the collective decision. It helps members of the crowd to find themselves in the solution, as everyone actually contributed a unique part of it, regardless how small it is and what role they have. This feeling of ownership will help to enact the decisions more effectively as the crowd feels more supportive to these decisions which are formed from their ideas and contributions.

\subsubsection{Increased relationship amongst the crowd.}

Managers stated that being different from each other reduces the competition amongst the crowd, while increasing their confidence, leading to more individual contributions to the acquired knowledge, and consequently supporting better relationships. Managers emphasised that management should provide a platform for "open and honest communication" and "respectful interaction" as prerequisites to make that work.

\subsubsection{Potential for conflict and clusters.}

Managers were cautious about managing diversity in collective decision making. Diversity could be seen as a cause of conflict, since different opinions may have conflicting characteristics and agendas. If not addressed properly and harnessed for richer diversity of perspectives and interpretations (Aroyo and Welty, 2013), this may lead to clusters of more powerful members and consequently minorities would be suppressed, leading to decisions that might create tension and reduced collaboration amongst the crowd.

\subsubsection{Management and coordination complexity.}

Another point to consider about diversity is that it may introduce high management and coordination overhead. As stated by a manager, this means that "the chairman must ensure that they capture the points and foster creativity," and it also 
means that "senior levels must not be allowed to bully other members". One of the strategies to prevent suppressed minorities is to "make quieter members of the team speak first so their views would not be overlooked by other team members who spoke louder". It does seem that management of diversity is highly dependent on personal skills of managers and would call for more systematic approaches, especially if we like to facilitate that by means of software.

Managers also expressed their views on the phases where applying diversity can be beneficial. They agreed that diversity is recommended in earlier stages of the decision-making and problem solving, while at later stages, a more centralised approach by senior members was recommended.

In their comments on the question about diversity, some managers also provided interesting insights about diversity assurance methods or mechanisms that they use within their enterprises to create and manage diversity. By analysing the comments, we could identify the following mechanisms:

- Letting it happen naturally: One manager stated that diversity in the crowd can be naturally created, "if you create the right culture". In their opinion, diversity cannot be fully specified and created and some degree of uncertainty would always be there whether the crowd is diverse enough. However, some aiding procedures in creating a culture which accepts and encourages diversity would lead to it happening in a spontaneous and natural way. In other words, diversity is seen as a result of culture and long-term procedures rather than deterministic and algorithmic approaches.

- Relying on intuitions: One manager said that to create diversity, they "do it by intuition". In their opinion, creating diversity was based on ad-hoc ways and not procedural, formal methods.

- Systematic sampling: Another manager used a kind of systematic sampling, gathering "at least one person from each level of the company, [and] giving each 'type' of staff a chance to input".

- Using project management methodologies: One manager explained that they followed "the procedures in PRINCE2 management regarding equality and diversity", therefore ensuring that "diversity training is undertaken and that the appropriate diversity strategies are followed". 


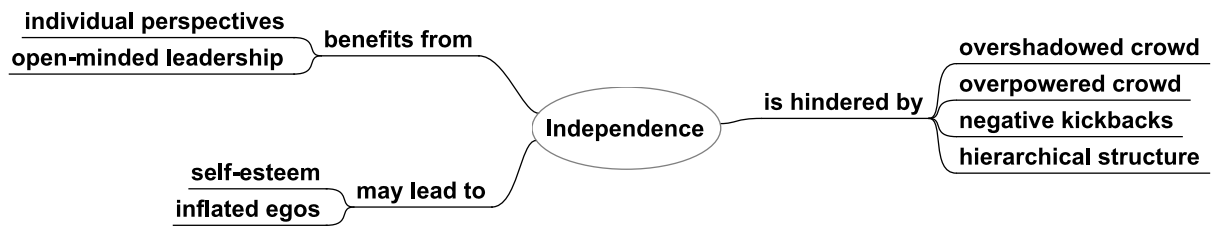

Figure 3: Mind map for Independence in the WoC

\subsection{Independence: The Second Principle of the WoC}

Independence of the members of the crowd is another principle of the WoC. It means freedom from the influence of others (Surowiecki, 2005). The main motive of independence is that individuals are more likely to identify a good decision if the individuals in the group think separately and are independent of each other's judgements. We collected managers' opinions on the concept of independence and how they view it in their practice of the WoC. Figure 3 shows a mind map of our findings in this section.

\subsubsection{Individual perspectives.}

Managers all agreed that members' own perspectives are essential to identify a good solution. They mentioned that it must be ensured that "each person is treated equally and that although there may be an 'expert' in the team, their opinion is as valid as another member of the group". Managers also observed that independence will also "allow members to feel in control of their own area of responsibility" and become more focused and accountable of what they say.

\subsubsection{Obstructing effects of overpowered or overshadowed crowd.}

Managers believed that being overpowered or overshadowed in a group could hinder the validity of group decisions. Therefore, they stated that it should be ensured that "dominant speakers are not allowed to overpower" and that they do not allow members to "stay quiet as they are shy or intimidated or do not think their opinion has value". This means a skilled moderator is needed and equity principles should be enforced.

\subsubsection{Open-minded leadership.}

Another decisive factor in independence was leadership. All managers agreed that having an open-minded leader would encourage members to say what they 
think regardless of their positions and expertise. Applying the WoC may look awkward when the management style is strictly centralised and hierarchical.

\subsubsection{Negative kickbacks.}

The managers also warned about the consequences of negative kickbacks, i.e. rejecting or ignoring suggestions coming from employees, even if they occur infrequently and not within the WoC context. Such practice would hinder the contribution of valuable and truthful knowledge from the crowd. They also mentioned that this should be consolidated by organisational processes and communication channels rather than being dependent on individual managers who would be occasionally subjective and biased. Being ad-hoc in communicating ideas would put the WoC at risk.

\subsubsection{The fine line between self-esteem and inflated ego.}

Managers said they always try to boost self-confidence and self-esteem of their staff and clients, as this will encourage them to speak more and also discover themselves more. However, managers also agreed that independence could expedite egos, with "some people trying to bully their opinion upon the group". Such selfishness may hinder the desired efficiency at the aggregation level as well ( $\mathrm{Na}-$ matame, 2006). Also, improperly managed independence may lead to a situation where members might forget that they are a part of a team and that reaching consensus requires receptiveness and negotiation. In multi-cultural enterprises, this could become a serious issue. As a result, training and workshops on diversity and cooperation values should be a prerequisite.

\subsubsection{Hierarchy.}

Managers agreed that hierarchy could affect what people report and say, but it could not affect what they think. Managers need to "ensure that staff understood that they were in a safe space" and that the managers "are not necessarily looking to the most senior members to take a leading role in the decision making", otherwise some may expedite what those senior members would say and contribute nothing. Although hierarchy is inevitable, it has to be kept to the minimum when applying the WoC, so that all members feel it is about the enterprise quality.

\subsection{Decentralisation: The Third Principle of the WoC}

Decentralisation means that "power does not fully reside in one central location", and that "many important decisions are made by individuals based on their own local and specific knowledge rather than by an omniscient and far-seeing manager" (Surowiecki, 2005). It can be seen similar to the divide-and-conquer 


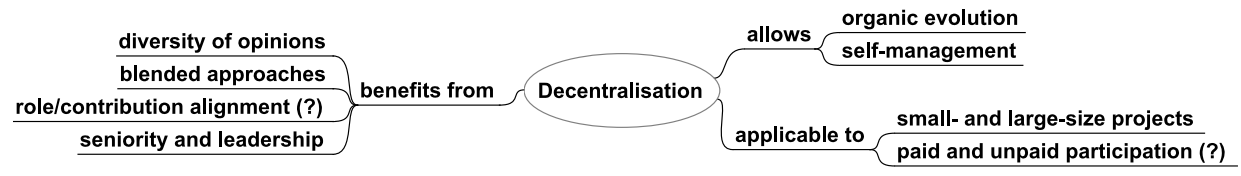

Figure 4: Mind map for Decentralisation in the WoC

strategy where members work on specific parts of the problem and contribute their local knowledge. We asked managers to give their perspectives on decentralisation. In contrast to the cases of diversity (Section 3.2) and independence (Section 3.3), decentralisation seemed to be a more arguable concept. Figure 4 shows a mind map of our findings in this section.

\subsubsection{Decentralisation and diversity of opinions.}

Managers' views were scattered as whether decentralisation is needed only when diversity of perspectives and opinions on the same subject is needed. While some managers believed that without diversity, there would be no need for decentralisation, other managers held the opposite view. It could be interpreted that other factors are also definitive in deciding whether such relationship exists or not. Culture, project size, crowd features, etcetera could be amongst these decisive factors. Managers also maintained that decentralisation "frees individuals to think in new ways or to apply their thinking to new paradigms".

\subsubsection{Project size.}

Managers disagreed with the notion that decentralisation is needed only in big projects where no one can have a complete view of the problem under discussion and the solution should be of a composite nature. They thought decentralisation could also play a role in medium and small-scale projects, perhaps because of the ease of coordination and the possibility to find domain experts who can see the whole picture.

\subsubsection{Paid vs. unpaid crowd.}

Managers had doubts whether decentralisation is a model which suits charities and free projects only, or it suits commercial companies too. This originates from the notion of responsibility, which may be enforced in for-profit environments where a high degree of commitment is expected from the paid participants. This is, however, not necessarily the case in non-profit organisations where participation 
is highly voluntary. This may mean missing certain pieces of information when forming the overall decision.

\subsubsection{Organic evolution.}

Managers believed that decentralisation allows projects to grow organically. This is made possible since the WoC prevents from dependency on a central reference point. The group dynamics may lead to emergent properties similar to what happens in a natural evolution. The different perspectives and the localised knowledge help the enterprise to cater for changes as seen by different actors.

\subsubsection{Blended approaches.}

Managers favoured both decentralised and centralised approaches at the same time. There was a consensus that a blended approach may sometimes be needed to harness the power of decentralisation, and also to assure that solutions are going to be found in a systematic way, and no delay will happen or no information will be missed. It is agreed that the intervention of a centralised authority has to be designed carefully and this depends on the context, e.g. whether the problem is critical and how it is seen by the different actors who provide their local knowledge. Other contexts within an enterprise, such as task uncertainty, may also need to be considered when deciding on adoption of a centralised or decentralised approach (Kim and Burton, 2002).

\subsubsection{Self-management.}

Some managers stated that decentralisation makes the management easier and also makes the changes smoother. In the voluntary decentralisation, "the staff members can easily feel out of their comfort zones". A cycle of brainstorming and decentralisation makes it "easy to identify areas of the team that are struggling earlier on and help iron out issues early before they become unmanageable". On the other hand, other managers mentioned that such self-management causes difficulties because "people work on a different pace and also miscommunication happen[s] all the time".

\subsubsection{Role-contribution alignment.}

Managers had varying ideas as to whether the WoC should ensure an alignment of what people contribute with their role in the enterprise. Some believed that this should be enforced, and that decentralisation should not mean uncontrolled contribution in that sense. Others thought that any member of the crowd should be entitled to voice their opinions about any issue, even if it is not aligned with their expertise or their role in the enterprise. Managers also did not see it as a 


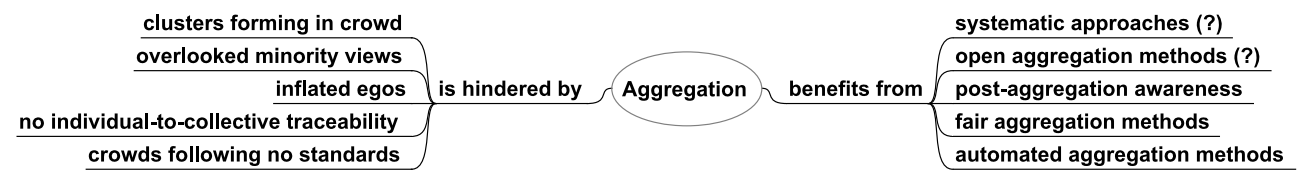

Figure 5: Mind map for Aggregation in the WoC

precondition that such roles and jobs should be held long enough before members can contribute as they thought they would typically need both fresh and expert staff knowledge.

\subsubsection{Seniority and leadership.}

Managers observed that seniority and having a person to manage the solutions are essential for a successful implementation of decentralisation. On the other hand, they expressed that good leadership should be seen as the secondary factor, and not the primary one, for identifying a good solution.

\subsection{Aggregation: The Fourth Principle of the WoC}

Aggregation provides a mechanism for turning people's private judgements into a more rounded collective decision (Surowiecki, 2005). Aggregation enables the assembly of variables into well-organised categories and summarises them to produce a collective judgement. When a group of self-governing individuals try to solve a problem, aggregation is the key to bring their efforts together. A badly-designed aggregation process is one of the main reasons for the failure of the WoC. Aggregation failures, however, may be due to the bad design of other principles too. We asked the managers on how they view and apply aggregation in their enterprises. Figure 5 shows a mind map of our findings in this section.

\subsubsection{Systematic approaches and automated support.}

Managers had two different opinions in this regard. Some admitted that the aggregation approaches lack rigour and they depend much on judgements of senior management. Other managers wondered if there could ever be such a systematic approach, as it is the case of artificial systems, e.g. sensors and multi-agent systems where the software is designed the way the designers prefer. The argument is that people typically like to express knowledge in a non-uniform manner and restricting them to a certain style will hinder the applicability of the WoC altogether. 
In both cases, managers agreed that an automated support will be beneficial and yet it is lacking.

\subsubsection{Open vs. controlled aggregation.}

Two different views were observed here. Some managers held the notion that aggregation should not be based on a predefined method and agenda. This means that it should adopt an open approach, e.g. grounded theory approach, otherwise bias will be introduced and the result will not reflect the real voice of the crowd. The other direction was that the WoC should be set up since the beginning to make aggregation work systematically; otherwise we cannot guarantee it yields quality, or even correct, aggregated results.

\subsubsection{Post-aggregation awareness.}

Managers stated that people typically contribute knowledge to the enterprise, but "they are typically unaware how it is aggregated to form the final decision". In certain enterprises, this could lead to reduced trust and transparency. Therefore, providing feedback to the crowd by telling them how their opinions were reflected in the final decision seems indispensable. However, and given that aggregation is not necessarily algorithmic, finding that relationship between the individual opinions and the collective decision is not always possible.

\subsubsection{Fairness and satisfaction being hard to achieve.}

Managers observed that aggregation could also face difficulties while dealing with inflated egos, i.e. certain members with big egos could be unsatisfied even if the aggregation was fairly conducted (regardless of how we define fairness here). While this is natural in any democratic-like system, some managers still see that aggregation may utilise weights for each member's voice in addition to percentages. Once more, this is still debatable and it is not clear when that would be a good option and whether this would go with the remit of the WoC.

\subsubsection{Automated mechanisms for aggregation.}

Managers specified different methods of aggregation for the information they obtained within their enterprises as a result of applying the WoC. One manager expressed percentage as a basic mathematical mechanism for aggregation. another manager proposed a weighing mechanism for members' opinions. He stated that "there are members of the team you would trust more than others so their opinions carry more weight". Furthermore, another manager proposed a scoring system "to place each idea on a scale and then explore the top three ideas in detail to find the solution". A final observation was that what the crowd says might not be uniform 
and would require normalisation. For example, for certain reasons, certain members may perceive very good as the highest possible score, while others may have no problems in viewing excellent as the highest score. However, real case studies might be needed to better identify all possible ways of aggregation when the WoC is applied within an enterprise. For example, a fuzzy mean could probably better suit the aggregation function than a percentage for certain fuzzy terms. That is, if eight lows, five mediums, and six highs were collected from the crowd as an answer to a certain question, a probable final aggregated answer could be medium (the fuzzy mean) instead of low (the higher percentage). The lack of algorithmic and concrete approaches makes the automation of aggregation either impossible or ad-hoc. The clarity in specifying how aggregation should be performed and the various styles of aggregating individual opinions to form a collective one will be the stepping stone towards developing CASE tools which can aid managers in the process.

Managers indicated an interesting issue about aggregation, stating that it could sometimes be deceiving, as certain members might end up shaping decisions which could be clearly against certain interests and opinions, including those belonging to them. When this is realised, collaboration will be reduced and then clusters within the crowd will start to appear. This will make it hard to reach a consensus or a collectively accepted opinion. Thus, the challenge is to have a precautionary procedure to make the aggregation progress and process transparent early on. Again, this might not be possible if aggregation is done at the last stages where experts will evaluate all gathered data and will take a decision.

\section{Discussion}

In Section 3, we reported and reflected on the opinions of the managers about the current practices of the use of the WoC within enterprises, and the four principles of the WoC. In this section, we discuss some more challenges and issues related to these four principles and we also provide a checklist for the analysis and design of WoC support tools. The following discussions are reflections and elaborations on the concept of WoC which are informed by the managers' answers. To back up our reflections and also to acknowledge some literature which shared those observations, we will also refer to the existing literature when appropriate.

\subsection{Observations on the Four Principles}

Catering for diversity remains a big challenge in the utilisation of the WoC (Fogg, 2008). The challenge arises because the meaning of diversity can change 
from one enterprise to another, and from one implementation of the $\mathrm{WoC}$ to another. In a related study, (Hosseini, et al. 2014) have identified four possible ways of making a crowd diverse. These four types of diversity are gender diversity, age diversity, location diversity and expertise diversity. However, it remains unclear how many of these four aspects might be needed to ensure the type of diversity that an enterprise is really looking forward to achieving. Furthermore, enterprises may need to have their own perceptions of some of these diversity types. For example, for one enterprise, location diversity might mean that they choose different employees from different departments, while for another enterprise, location diversity might mean that they choose employees within the same department but from different branches. Therefore, the interpretation of diversity and ensuring it remains highly dependent on its usage and policy makers' perception.

Another challenge concerning the diversity lies not on ensuring the diversity of the crowd, but on ensuring the diversity of the answers obtained from them. In essence, it is sometimes the case that by introducing diversity in the crowd, we really need diverse and varying answers and solutions. This means that under these circumstances, it is not the diversity in the crowd that is an enterprise final goal, but it is the diversity in the ideas, opinions, responses, innovations, etcetera that the enterprise is actually seeking. This has already been noticed in some works in the field of the WoC, such as (Lasecki, et al., 2014), but this study is focused on a single case study, and therefore cannot be generalised to all problems within the domain of the WoC. This challenge, consequently, calls for more study about diversity of solutions or opinions as opposed to diversity of individuals.

It should be stated that despite all the shortfalls in ensuring diversity during the utilisation of the WoC, it remains a fact that enterprises with diverse employees often perform best, and it is worth spending money on diversity training, education, and outreach (Page, 2007).

Ensuring the independence of the individuals within the crowd can be challenging as well. When the individuals are asked for their opinions separately, e.g. via online questionnaires or one-to-one interviews, their independence of thought can usually be guaranteed to a high extent. On the other hand, it is very difficult, if not impossible, to guarantee such independence of thought in focus groups, business meetings, etcetera, when a group of people are asked for their opinions in the presence of other members of the crowd. Group characteristics may lead to biases in logical thinking, such as group polarisation (Janis, 1982) and representativeness fallacy (Argote, et al., 1999). For example, (Lorenz, et al., 2011) show that even a slight social influence can undermine the WoC effect in simple estimation tasks. Although such a social influence can sometimes prove to be useful, as shown by 
(Farrell, 2011), preventing those adverse social effects on the independence of individuals, at least in some cases, is not manageable by other authoritative figures and is more related to personal characteristics of the individuals.

Another challenge concerning the independence of the individuals in the WoC is that there are no metrics upon which such independence can be measured. Every individual is affected by the public opinion or peer pressure to some extent, and upon applying the $\mathrm{WoC}$, it cannot be measured whether every individual's opinion is previously affected by some other individual's viewpoints or not, and if yes, how much it is influenced by it. It seems that more studies should be conducted in this regard.

Reaching decentralisation in an enterprise needs courageous decisions on managers' and policy makers' sides. Bestowing power to individuals and spreading the decision points within an organisation means that managers must begin to trust in their employees, otherwise "any opening-up of innovation is absurd" (Collm and Schedler, 2012). However, as in any trust-based relationship, this one-sided trust is not sufficient. It needs to be both-sided, meaning that the employees should also trust in the enterprise (Collm and Schedler, 2012). Only then such decentralisation will achieve its goals (Olleros, 2008), otherwise it may lead to adverse effects on the enterprise.

Decentralisation itself also encounters cultural barriers. In societies where decision making is generally centralised, it is rather difficult to implement decentralisation within an enterprise. This is because the culture of following the central authority and dependence of thoughts are deep-rooted within individuals. It might be that decentralisation in such environments will yield no obvious benefits to the enterprise, and even lead to less productivity and more difficulties in management.

Aggregation methods for the $\mathrm{WoC}$, besides the ones that were discussed in the previous section, have been studied in several studies, for example in (Baharad, et al. 2011) and (Bachrach, et al., 2012). These studies and similar ones do actually identify metrics and methods for aggregation. It is different from the other three principles in the $\mathrm{WoC}$, where no metrics are provided. This is probably because aggregation of different opinions and solutions needs to be dealt with in every project where the WoC is applied, and therefore metrics should be provided for aggregation in practice. On the other hand, the proposed aggregation methods are based either on managers' experiences within their enterprises or based on scholars' case studies. We still lack an engineering approach to provide us with an appropriate aggregation method which suits the context and the problem at hand.

Another interesting issue regarding aggregation is the link between an individ- 
ual's feedback and the final aggregated decision. More studies need to be done on the traceability of every individual's input and the aggregated output, as this can motivate those individuals and can also give them a sense of usefulness within the enterprise.

Finally, another issue in the domain of WoC is automated support. At the moment, automated support is not being widely applied even for tasks for which we have theoretical solutions. Only general purpose tools are being used now and there is a general lack of designated tools tailored specifically for the WoC in the current practice of enterprises involved in our study. For example, document sharing and commenting and general purpose forums such as social media are being used in order to gather opinions. In addition, automated support is probably a less challenging problem when we first succeed to develop solid theoretical foundations, e.g. for enhancing aggregation, assuring decentralisation and eliminating bias.

\subsection{A Checklist for the Analysis and Design of WoC-Support Tools}

Our study concluded that there is a lack of automated support to the process of the WoC. Managers expressed the need for that, especially for medium-scale and large-scale projects. In this section, we highlight the challenging design choices in developing WoC projects and their governance by introducing a list of questions related to each WoC aspect. They are elaborated and deduced by the collective analysis of the findings of the interviews and also by the percentages and comments in the survey phase. Answering these questions in the early stages of development will result in increasing the usability and efficiency of such an automated support.

\subsubsection{Diversity}

- What are the set of skills, roles, and experience needed for the problem?

- What will be the mechanisms used to let the crowd recognise their uniqueness in comparison to the rest?

- What will be a mechanism to mitigate conflicting viewpoints?

- When is it suitable to seek for diversity and when to start centralising the process?

- When and how will management need to intervene in the coordination to allow all different perspectives to appear? 
- What are the appropriate metrics to create and manage diversity in the crowd?

\subsubsection{Independence}

- What selection criteria will be applied to ensure that recruited members have their own perspective and how should it be sustained when interaction starts?

- What countermeasure and precautionary procedure should be applied to prevent overshadowing certain members or groups?

- What interaction styles will be adopted to ensure effective leadership and to avoid negative kickback?

- What is the preventive and corrective process to deal with inflated egos in the crowd?

- How would the system ensure that seen or felt hierarchy does not affect trustworthiness of opinions stated especially by the crowd in the lower levels of the pyramid?

- What are the appropriate metrics to create and manage independence of individuals in the crowd?

\subsubsection{Decentralisation}

- Which parts of the project require decentralisation and distributed local knowledge?

- What will be the different roles to which the crowd will be allocated and what will be the criteria for such allocation?

- When the crowd participates on a voluntary basis, what will be the procedure to ensure that trustworthy local knowledge is provided in a timely manner?

- How is decentralisation going to be enacted if the WoC is planned as a lifelong activity and iterative evolution of the decisions?

- How will the switch between centralised and decentralised stages be enacted?

- What are the appropriate metrics to create and manage decentralisation? 


\subsubsection{Aggregation}

- Will aggregation follow a grounded theory approach, i.e. based on interaction and emergent properties, or will it follow predefined workflow, and how?

- How can the crowd see how their contribution is reflected in the final decision?

- Should the crowd see the intermediary steps of the aggregation, i.e. when the data collection is still on, and how?

- What mechanisms will be used to quantify the crowd opinions when calculating the aggregated decision takes place?

- How should it be measured whether the final decision is a collective one?

- What are the appropriate metrics to create and manage aggregation of information obtained from the crowd?

\subsection{Threats to Validity}

Our study has a number of threats to validity. We explored the experience of applying the WoC from the perspective of the managers. Asking employees would perhaps lead to a more holistic result. Furthermore, although we used purposeful sampling for both phases, it is hard to precisely specify how much expertise each of the participants got in applying the WoC, especially that many said they apply it under different names. Some statements were also debatable, e.g. whether aggregation should be open or controlled. We expect that further analysis will lead to a clearer picture. However, these debates are themselves a good result as this points out what areas of research to explore in future work. Two out of 27 participants in the survey phase stated that they did not believe in the WoC and did not apply it in practice, but they had seen it being applied. Although this makes their answers speculative, their comments were interesting and we used that to raise some debates, e.g. those in decentralisation and aggregation.

\section{Conclusion}

In this study, we explored the managerial perspective of the concept of the WoC, its practices and its challenges. 35 managers from 33 UK enterprises participated in both phases of our study. We observed that a vast majority of experts agreed on the need for the concept and also a shared understanding of the role of 
diversity and independence in the crowd. However, some debates about decentralisation and aggregation were found. We concluded that there is still a lack of automated support of the process and a heavy reliance on the personal skills of senior management, especially in the aggregation stage. While the results might not be generalisable to work environments at the world level, a high degree of decentralised and non-hierarchical nature can be perceived as a common norm in several UK enterprises. This can make the results generalisable to other similar work environments. Our future work will try to concretise more the four principles of the WoC, as this will be an essential preliminary step to have such an automated support.

\section{Acknowledgements.}

The research was supported by an FP7 Marie Curie CIG grant (the SOCIAD Project) and by Bournemouth University through the Fusion Investment Fund (the BBB, BUUU and VolaComp projects) and the Graduate School Santander Grant for PGR Development.

\section{References}

A. Collm, K. Schedler: Managing crowd innovation in public administration. International Public Management Review, 13(2), pp 1-18 (2012)

A. Fuxman, P. Tsaparas, K. Achan, R. Agrawal: Using the wisdom of the crowds for keyword generation. Proceedings of the 17th international conference on World Wide Web, pp 61-70. ACM (2008)

A. Kittur, R.E. Kraut: Harnessing the wisdom of crowds in wikipedia: quality through coordination. In: Proceedings of the 2008 ACM conference on Computer supported cooperative work, pp 37-46. ACM (2008)

A. Namatame: The wisdom of networked evolving agents. Advances in Artificial Economics, pp 149-165. Springer Berlin Heidelberg (2006)

A.E. Murr: Wisdom of crowds? A decentralised election forecasting model that uses citizens local expectations. Electoral Studies, 30(4), pp 771-783 (2011)

B.W. Nocco, R.M. Stulz: Enterprise risk management: theory and practice. Journal of Applied Corporate Finance. 18, pp 8-20 (2006) 
E. Baharad, J. Goldberger, M. Koppel, S. Nitzan: Distilling the wisdom of crowds: Weighted aggregation of decisions on multiple issues. Autonomous Agents and Multi-Agent Systems, 22(1), pp 31-42 (2011)

E. Motta: 25 years of knowledge acquisition. International Journal of HumanComputer Studies, 71, pp 131-134 (2013)

F.X. Olleros: Learning to trust the crowd: Some lessons from Wikipedia. International MCETECH Conference on E-Technologies, pp 212-216. IEEE (2008)

H. Oinas-Kukkonen: Network analysis and crowds of people as sources of new organisational knowledge. Knowledge Management: Theoretical Foundation. Informing Science Press, pp 173-189 (2008)

I.L. Janis: Groupthink: Psychological studies of policy decisions and fiascoes. Boston: Houghton Mifflin (1982)

J. Barjis, G. Kolfschoten, A. Verbraeck: Collaborative enterprise modeling. In E. Proper, F. Harmsen, J. Dietz, eds.: Advances in Enterprise Engineering II. Volume 28 of Lecture Notes in Business Information Processing. pp 50-62. Springer (2009)

J. Kim, R.M. Burton: The effect of task uncertainty and decentralization on project team performance. Computational \& Mathematical Organization Theory, 8(4), pp 365-384 (2002)

J. Lorenz, H. Rauhut, F. Schweitzer, D. Helbing: How social influence can undermine the wisdom of crowd effect. Proceedings of the National Academy of Sciences 108(22), pp 9020-9025 (2011)

J. Pierre: Debating governance: Authority, steering, and democracy. Oxford University Press (2000)

J. Surowiecki: The wisdom of crowds. Random House LLC (2005)

J. Surowiecki, M.P. Silverman, et al.: The wisdom of crowds. American Journal of Physics. 75, pp 190-192 (2007)

J.W. Creswell, V.L.P. Clark: Designing and conducting mixed methods research.(2007) 
L. Argote, R. Devadas, N. Melone: The base-rate fallacy: Contrasting processes and outcomes of group and individual judgment. Organizational Behavior and Human Decision Processes, 46(2), pp 296-310, (1999)

L. Aroyo, C. Welty: Crowd Truth: Harnessing disagreement in crowdsourcing a relation extraction gold standard. WebSci2013. ACM (2013)

M. Hosseini, K. Phalp, J. Taylor, R. Ali: The four pillars of crowdsourcing: A reference model. IEEE Eighth International Conference on Research Challenges in Information Science (RCIS), doi: 10.1109/RCIS.2014.6861072. pp 1-12 (2014)

M. Vukovic: Crowdsourcing for enterprises. In 2009 World Conference on Services-I, pp 686-692. IEEE (2009)

M.Q. Patton: Qualitative research. Wiley Online Library (2005)

P. Fogg: Catering to a diverse crowd. Chronicle of Higher Education 55(5), (2008)

R. Baeza-Yates: Wisdom of crowds or wisdom of a few? Web Engineering, 573 (2014)

R.G. Curty, P. Zhang: Social commerce: Looking back and forward. Proceedings of the American Society for Information Science and Technology. 48, pp 1-10. Wiley Online Library (2011)

R.V. Kozinets, A. Hemetsberger, H.J. Schau: The wisdom of consumer crowds collective innovation in the age of networked marketing. Journal of Macromarketing. 28, pp 339-354 (2008)

S. Farrell: Social influence benefits the wisdom of individuals in the crowd. Proceedings of the National Academy of Sciences, 108(36), 625 (2011)

S.E. Page: Making the difference: Applying a logic of diversity. The Academy of Management Perspectives, 21(4), pp 6-20 (2007)

W.S. Lasecki, C.M. Homan, J.P. Bigham: Tuning the diversity of open-ended responses from the crowd. arXiv preprint arXiv:1408.6621 (2014)

X. Jin, A. Gallagher, L. Cao, J. Luo, J. Han: The wisdom of social multimedia: using flickr for prediction and forecast. In: Proceedings of the international conference on Multimedia, pp 1235-1244. ACM (2010) 
Y. Bachrach, T. Graepel, G. Kasneci, M. Kosinski, J. Van Gael: Crowd IQ: Aggregating opinions to boost performance. Proceedings of the 11th International Conference on Autonomous Agents and Multiagent Systems - Volume 1 (AAMAS '12), Vol. 1. International Foundation for Autonomous Agents and Multiagent Systems, Richland, SC, pp 535-542 (2012) 